\title{
Piloting Undergraduate Student-led Health Check Service
}

There is a growing emphasis on developing communication and clinical skills during undergraduate pharmacy programmes, especially within the recent General Pharmaceutical Council consultation on initial education and training [1]. A health check service delivered by pharmacy students has shown benefits in the development of such skills and students found it as good as more traditional learning activities [2]. Additionally, it can be successfully incorporated within the curriculum within the United Kingdom context [2,3].

Our own exploratory work at the School of Pharmacy and Medical Sciences (SPMS), University of Bradford, identified that our pharmacy students strongly desired for more practical learning experiences in order to feel better prepared for their first day of professional practice [4]. Additionally, SPMS staff consultation confirmed that students from Year 3 should have the knowledge and skills to deliver the tests required of a health check [5]. Therefore, building on our previous work and valuable experience from Catherine Langran and colleagues at the University of Reading, we piloted a student-led health check service to the University's community. The pilot service was delivered by six undergraduate pharmacy students as part of their self-selected research project.

The service, including standard operating procedures, was designed by adapting the national health check service framework and work from the University of Reading. Following ethical approval, third year pharmacy students attended two training sessions, each lasting two hours: 1) practical skill training to perform health check tests, and 2) competency assessment, which students were required to pass. Students were also made aware of the risks associated with handling clinical waste (e.g. lancets used for finger pricking) and how to minimise those risks.

The student-led service was advertised on the University's website in September 2018. The service received high interest and all appointment slots were filled within three days.

Students worked in pairs over three days in October 2018 at the Digital Health Zone Enterprise Academic building at the main campus. This multi-disciplinary facility houses: physiotherapy and optometry public clinics, health promotion areas, and digital diagnostics. The health check service was hosted within our medicines review hub, which has private consultation rooms. One or two supervisors (registered and practicing pharmacists) were available at all times to offer advice to the students and review overall health check results. Tests performed included: height and weight measurement, body mass index calculation, waist and hip measurements, body fat composition, blood pressure and pulse readings, physical activity and lifestyle assessment, and $\mathrm{QRISK}^{\circledR} 3$ calculation where appropriate. Supervisors performed cholesterol and blood glucose tests. Participants were able to opt out of any test. Students interpreted the results and discussed with supervisors before offering lifestyle advice, health promotion literature and setting healthy living goals with the 
participant. Each participant was provided with a personalised copy of their test results and asked to complete a feedback form.

Thirty-eight participants, 28 staff members and 10 students, from across the University attended the service. More females $(n=23)$ than males participated and the average age of participants was 40 (range $20-62$ years). Twenty-six (68\%) participants had not had a health check before and $14(37 \%)$ indicated to have at least one long-term condition. Health checks lasted on average 41 minutes (range 18-60 minutes).

All participants were offered tailored healthy lifestyle information and were encouraged to set a goal. Thirty-seven (97\%) participants were provided information on healthy diet and increasing physical activity due to: BMI being overweight, high waist to hip ratio, and/or elevated cholesterol readings. No participant required referral to their general practitioner.

The student-led service was well received by the University's community and pharmacy students themselves. Majority of participants, $n=36(98 \%)$, stated they were very likely to recommend the service to a colleague and attend again if the service was run regularly on the campus ( $n=34,89 \%)$. Many participants $(n=33,87 \%)$ stated they would make changes to their lifestyle because of the health check. The pharmacy students valued the opportunity to try advanced communication skills, including the principles of coaching, and applying their theoretical knowledge into practice through real patient contact. Recommendations from participants for improvement included easier booking processes, clearer location signposting and more instructions before the appointment e.g. what to wear.

We have demonstrated that students can deliver a health check service that has value for participants and contributes to the development of students' confidence in undertaking clinical activities. Future work will focus on incorporating this service within our current pharmacy undergraduate programme, allowing all students the opportunity to take an active role in service provision. However, further considerations are required for delivering student-led clinical activities to local communities. These include (but not limited to) timetabling, supervision, and governance, including professional liability with regards to appropriate insurance. There is currently limited guidance and we should learn from others who are successfully running student-led clinics to members of public e.g. physiotherapy, optometry, dentistry.

Kristina Medlinskiene and Justine Tomlinson, Pharmacy Doctoral Training Fellows, University of Bradford 
1. General Pharmaceutical Council (2019). Consultation on initial education and training standards for pharmacists. Available at:

https://www.pharmacyregulation.org/sites/default/files/document/consultation_on_init ial_education_and_training_standards_for_pharmacists_january_2019.pdf [Accessed 14 January 2019].

2. Langran $C$ and Alexander A. (2012). Can an On-site Clinical Experience Be as Good as or Better Than Learning from Lectures, PBLs, Workshops and External Placements? Research in Social and Administrative Pharmacy 8(6):e13-e14.

3. Langran C, Hannan R, Greenland B, and Donyai P. (2017) Outreach activity of healthy living assessments provided by pharmacy students to the local community. International Journal of Pharmacy Practice Supplement 1:43-44.

4. Tomlinson J, Yaqoob U, Shabbir S, Medlinskiene K. (2018) Exploring the use of digital technology in the MPharm programme to prepare students for their first day of practice. Journal of Pharmacy Education 18(1):e231-e254.

5. Medlinskiene K, Tappas T, Tomlinson J (2018) What health-related activities could be delivered by pharmacy students in the Digital Health Enterprise Zone (DHEZ) Academic? Journal of Pharmacy Education 18(1):e231-e254. 